\title{
REPRESENTACIONES DE GÉNERO EN LAS PUBLICACIONES PERIÓDICAS DE NICARAGUA (1950-1979): ANÁLISIS HEMEROCRÍTICO ${ }^{10}$

Nohemí Rojas Icabalzeta ${ }^{11}$

Representaciones de género en las publicaciones periódicas de nicaragua (1950-1979): un análisis hemerocrítico

Resumen: Los años 50, 60 y 70 marcan una coyuntura en la historia de Nicaragua, también desde la perspectiva de género. En esta investigación se recogen acontecimientos con relación a las representaciones de género manifiestas en los dos diarios con mayor circulación a nivel nacional en la época: Novedades y La Prensa; el primero manejado por la familia somocista; y en consecuencia, el siguiente asumía un papel opositor en contra del gobierno imperante. El recorrido por las páginas de La Prensa y Novedades en el contexto de 1950 a 1979, ofrece un análisis integral en relación a la concepción de la mujer en aquella época. Esta visión de está manifiesta a través del estudio de categorías como los estereotipos de género que modelan patrones de comportamiento, las clases sociales impuestas por la manipulación mediática, la concepción de belleza femenina y la recepción de los mensajes de la población de la época en estudio.

Palabras clave: Representaciones de género, publicaciones periódicas, análisis hemerocrítico, estereotipos, manipulación mediática, clases sociales, belleza.

Gender Representations in newspapers in Nicaragua (1950-1979): An Hemerocritic Analysis Abstract: The 50s, 60s and 70s mark a turning point in the history of Nicaragua and in the case of gender. In this article, we deal with events related to gender representations in the two largest newspapers in those decades: Novedades and La Prensa; the former handled by the Somoza family; and the latter departing from a stanza of opposition to the prevailing role government. The journey through the pages of La Prensa and Novedades in the context of 1950-1979, offers a comprehensive analysis related to the conception of women at that time. This view of women is manifested through the study of categories like gender stereotypes that shape behaviour patterns, social classes imposed by media manipulation, the conception of female beauty and the reception of messages from the population at the time in study.

Keywords: Representations of gender, periodicals, hemerocritical analysis, stereotypes, media manipulation, social classes, beauty.

\footnotetext{
${ }^{10}$ Fecha de recepción: 12/09/2016.

Fecha de aceptación: 24/11/2016.

${ }^{11}$ Docente del área de Investigación de la Facultad de Humanidades y Ciencias Jurídicas de la Universidad Nacional Autonóma de Nicaragua en Managua, Nicaragua; $₫$ nahomyrojas21@ gmail.com. Esta investigación ha sido financiada por el Fondo para Proyectos de Investigación (FPI) a través del Vicerrectorado de Investigación Dirección de Investigación de Postgrado de la Universidad Nacional Autónoma de Nicaragua, Managua (UNAN- Managua) quien merece mi agradecimiento por su colaboración en este ejercicio académico.
} 


\section{Introducción}

Las representaciones de género han estado inmersas desde siempre. El periodo de la dictadura somocista en Nicaragua (1937-1979) es una de las etapas históricas más estudiadas desde diversas disciplinas del saber. El largo período que duró este régimen marcó varias generaciones, cuyos remanentes todavía se manifiestan de alguna manera. Sin embargo, las nuevas generaciones de los ochenta y noventa no vivieron la época, únicamente la han conocido por medio de las narraciones de sus padres o abuelos; otros, por lecturas de periódicos o libros que recogen los acontecimientos históricos.

El análisis del discurso periodístico en dos de las publicaciones más destacadas de la época: La Prensa y Novedades, permite establecer el tipo de representación de la mujer que el régimen político construyó. En consecuencia, el objetivo general del estudio es analizar las representaciones de género manifestadas en las publicaciones periódicas de Nicaragua (1950-1979), a través de un análisis hemerocrítico, comprendiendo que los medios de comunicación modelan patrones de comportamientos y establecen estereotipos de género.

En el desarrollo de la investigación se estudian de manera integral los elementos: estereotipos, clases sociales y belleza, partiendo del postulado del filósofo e historiador Ken Earl Wilber, quien propone el análisis holístico para comprender la realidad social a partir de cuatro cuadrantes: conciencia, cerebro, estructuras sociales e imaginario colectivo, en el que el todo es más que la suma de sus partes, esto con el fin de crear un panorama completo referente al contexto de la mujer en los años 50, 60 y 70.

El estudio se inscribe dentro del enfoque de investigación cualitativo de tipo descriptivo; por tanto, se aplicaron distintos métodos concernientes a esta línea. El sustento teórico se realizó mediante el análisis documental, análisis hemerocrítico y la técnica de las fuentes orales. En relación a la selección y muestra periodística de los medios de comunicación, se combinaron factores y circunstancias que caracterizan el periodo en estudio como una coyuntura en la historia de las mujeres en Nicaragua. En los años 50, 60 y 70 el medio de comunicación por excelencia era la prensa escrita, la radio y la televisión no se habían desarrollado sino hasta en la década de los 70. Por tanto, la 
comunicación entre la sociedad de esa época se establecía a través de los periódicos. El diario Novedades era la voz oficial del gobierno de Somoza, en respuesta a la misión de controlar las empresas de comunicación en Nicaragua. Por su parte, La Prensa se caracterizó por su acérrima oposición al somocismo.

Los años 50 estuvieron marcados por un profundo interés de intelectuales y primeras feministas para luchar por los derechos legales y sociales de la mujer nicaragüense. El derecho al voto femenino en Nicaragua fue aprobado el 20 de abril de 1955 y el 3 de febrero de 1957 las mujeres votaron por primera vez. Por otro lado, 1979 también sella un hito en la historia de las mujeres y en los estudios sobre su participación social en Nicaragua. A partir de 1980 se generaron investigaciones acerca del papel de las mujeres en la vida social y en otros campos específicos de la política como su participación en la Revolución Popular Sandinista, cuando con su aporte, el 19 de julio de 1979 el pueblo logró abatir a Anastasio Somoza Debayle y con ello al régimen somocista.

En relación a la metodología para la revisión periodística, se seleccionó una muestra aleatoria por conveniencia de los periódicos por meses específicos concernientes a efemérides nacionales y mundiales, así como otros meses en los que se obtuvo hallazgos de importante data. Las revisiones de los diarios impresos se efectuó en la Bibliotecas: UNAN-Managua "salomón de la Selva", Banco Central de Nicaragua "Roberto Íncer Barquero" y Hemeroteca Nacional "Manolo Cuadra". El acceso al archivo hemerográfico fue limitado, en algunas situaciones por el mal estado de determinadas ediciones, y en otros casos ciertas publicaciones no se encontraron. Este particular, sin embargo, no impidió la búsqueda de las ediciones necesarias en otras hemerotecas en donde sí se logró tener acceso de algunas.

\section{Estereotipos de género y patrones de comportamiento: instauración de un régimen}

El término régimen político hace referencia al sistema político y social que rige un determinado territorio. Asimismo, denomina la estructura organizativa del poder 
político, con sus instituciones, normas y dirigentes. En el presente estudio se designa el término para el gobierno imperante de la época, así como para los medios de comunicación de análisis Novedades y La Prensa. En el caso del primero, como propiedad del estado somocista y el segundo constituido como un poder opositor al estado de ese entonces, y por ende, transmisor de ideologías en la sociedad de esas décadas, generando así patrones de comportamientos, los cuales se analizan mediante el contenido textual e iconológico de las publicaciones relacionadas con el papel de la mujer nicaragüense.

En el contexto de finales de 1969, el diario Novedades cumplía su función de posicionar en la opinión pública nicaragüense que el liberalismo, impuesto por la familia Somoza era símbolo de "Paz, Progreso y Libertad". Para este medio, fue propicio ilustrar su mensaje a través de la imagen de tres mujeres desfilando en una pasarela; debajo del escenario, un público de admiradores que complacientes veneran dichas virtudes femeninas. La imagen publicitaria vindicaría y legitimaría el accionar de la familia Somoza y su pensamiento liberal para fortalecer su régimen totalitario (ver figura 1).

El 30 de mayo de 1969 fue una fecha propicia para que a través del diario Novedades la primera dama, Esperanza Portocarrero de Somoza, emitiera un mensaje a las madres de Nicaragua a quienes delegaba el fortalecimiento de "la vida moral de las familias". Sin duda, un modelo que alude a la función de la mujer, quien debía mantenerse como base dentro de las familias y, por ende, de la sociedad (ver figura 2).

\section{Las labores femeninas, patrones de comportamientos desde los medios y la publicidad}

Los medios de comunicación generan patrones de comportamientos y aunados a la publicidad refuerzan conductas sociales y engendran fenómenos culturales. En las décadas en estudio era común apreciar imágenes de madres que satisfechas ejercen las labores del hogar y que transmiten su rol a próximas generaciones (ver figura 3). 


\section{Hacia la liberación femenina}

En la década de los 60 ya se avizoraban indicios de insatisfacción de las féminas en rechazo a los esquemas culturales. Esta liberación se manifestaba en tres aspectos: moda, autonomía de los cuerpos y la inserción de estas en espacios laborales. En 1969, los medios de comunicación divulgaban la noticia desde ciudad del vaticano, en Roma, de que el Papa VI, bajo un decreto ordenaba que las mujeres ya no cubrirían su cabeza con el velo o rebozo. Pero el periódico, junto a la nota de ámbito religioso, publicó otro hecho que causaba sensación en Nicaragua. Se trataba de los aclamados concursos de belleza. En esta ocasión se realizaba una pasarela en la ciudad de Granada relacionada con las minifaldas de las "jovencitas", a quienes se les calificaría por llevar "la más corta". El periódico denomina "una batalla del centímetro" y deja al lector como jurado de las participantes del concurso. (La Prensa, 3 de mayo de 1969)

Las mujeres nicaragüenses fueron reconocidas como ciudadanas en 1950, pero sin derecho a elegir y ser electas. "El sufragio ubica a los ciudadanos de cualquier sociedad en una posición de igualdad, pues al momento de una elección, tan importante y preciado es el voto de un profesional, un analfabeta, un rico, un pobre, un hombre o una mujer. De ahí que, alcanzar este derecho significó, para las nicaragüenses, una verdadera lucha por la igualdad". (Montenegro 2012: 92)

En su postulación para fungir como presidente de Nicaragua en el periodo 19671972, Anastasio Somoza Debayle acredita al Partido Liberal Nacionalista (PLN) la reivindicación de los derechos de la mujer (Somoza 1967: 156). Ciertamente, una de las limitantes para argumentar esta postura es la poca historiografía respecto al tema (ver figura 4).

\section{La mujer guerrillera y revolucionaria}

El rol de la mujer en la organización de las guerrillas y en el triunfo de la Revolución Popular Sandinista, significó un gran avance para la reivindicación de sus 
derechos y una importante contribución al sistema político y social de la época. Su papel de liderazgo femenino emergió de manera personal y colectiva.

Una de las páginas del periódico La Prensa de 1970, relata el hecho de dos mujeres campesinas detenidas por oficiales de la Guardia Nacional, estas fueron acusadas de ser guerrilleras. En este orden, la época implicaba una mayor dificultad, puesto que participar en movimientos armados era un delito doble para la mujer, quien ya tenía asignados sus roles de ama de casa y reforzadora de patrones morales de comportamientos en la sociedad (ver figura 5).

\section{Clases sociales y representaciones femeninas}

En este acápite es necesario destacar cómo el lenguaje clasista estaba homogeneizado en ambos periódicos. Así, términos como “doña”, "damas”, "elegante almuerzo", eran muy frecuentes para legitimar el estatus social femenino; era el caso de mujeres pertenecientes a las diferentes "Asociaciones de Damas", que se reunían en conmemoración de cumpleaños u otros eventos. De esta manera, los espacios para las mujeres estaban reducidos a labores benéficas y sociales. Así, pueden registrase actividades relacionadas a festivales en honor a la primera dama de la época, conmemoración de cumpleaños de la familia en el poder, entre otros temas triviales.

\section{Ideal de la familia}

Las representaciones de género en las publicaciones periódicas están íntimamente relacionadas, además del factor socioeconómico con el ideal de la familia. Esto es, la unidad familiar como base fundamental para el desarrollo de la sociedad. Cabe destacar, cómo la difusión del ideal de la familia en el imaginario colectivo se legitima a través de dos sistemas, por un lado, la iglesia como institución influyente en las personas, es esta la que determina en los sujetos sociales qué es lícito y qué no lo es desde el punto de vista religioso. El casamiento, los bautizos y, por ende, la unión familiar, son mandatos 
institucionalizados por la iglesia; "un buen cristiano" debe cumplir, por lo tanto, con esta orden.

\section{Arquetipos de belleza y cultura femenina}

Los arquetipos de belleza y cultura femenina que influenciaron en gran medida a la sociedad de las décadas de los años 50, 60 y 70 evidentemente estuvieron predeterminadas por los medios de comunicación, en estos también se inscriben el cine y la música. Estos elementos se fortalecieron en el imaginario colectivo a través de eventos como los certámenes de belleza, promociones de marcas, productos cosméticos y figuras icónicas del cine como Marilyn Monroe y otros arquetipos provenientes de las sociedades desarrolladas de Estados Unidos y Europa.

La década de los 60 en Nicaragua, al igual que el resto del mundo, estuvo marcada por grandes cambios en el plano de la moda femenina. La falda corta, con quince centímetros menos de la tradicional, fue una de las pautas que avizoraba la revolución sobre la visión de las mujeres en esta época. Estos cambios en los esquemas de percepción de las mujeres no se reducían únicamente al punto de vista estético de la moda, también abarcaron la concepción sobre el reconocimiento y validación de su propia condición en la sociedad (ver figura 6).

\section{Concursos de belleza}

$\mathrm{Si}$ los cambios vertiginosos en las nuevas tendencias de modas femeninas presentan transformaciones en las concepciones de belleza y el pensamiento en las décadas de los años 60 y 70 en Nicaragua, fue en gran medida por los certámenes de belleza. Las concursantes cumplían con los estándares preestablecidos: estatura promedia, carisma y hermosura, es decir, ni muy delgada ni con mucho peso.

El historiador José Salomón Delgado, respecto a la influencia de los arquetipos de belleza, refiere: "[...] siempre el modelo europeo agarra el modelo griego de la fineza de 
los contornos faciales, y las personas de recursos en Nicaragua, pues, la cultura norteamericana influyó mucho, eran sus modelos las actrices [...]” (Delgado 2016).

La estética de los cuerpos es un elemento que ha variado en cada tiempo histórico. La publicidad y los medios de comunicación han remarcado el modelo de belleza en cada época. En los años 50, 60 y 70 en Nicaragua las mujeres debían ser de contexturas "hermosas" y no muy delgadas. Sossa (s.f), añade: “[...] todavía hasta los años 50 la publicidad propone un cuerpo floreciente y lleno, las mujeres gruesas son las mujeres soñadas por los hombres y el modelo anhelado y soñado por las mujeres”. Igualmente, según dicho estudio, el estar con unos kilos de más era sinónimo de estar "sanito" (ver figura 7).

En su libro El mito de la belleza, Wolf (1992), plantea que la belleza no es universal ni inmutable, aunque Occidente pretenda derivar todos los ideales de belleza femenina de una especie de idea platónica. De acuerdo con Eco (2006), la belleza y su interpretación han cambiado constantemente con el tiempo, haciendo que se adapten y ajusten a los conceptos del momento. Este señala que los conceptos que se adaptan en cada época de nuestra historia determinan los estereotipos en los cuales los seres humanos nos basamos para determinar si algo es bello o no.

\section{Recepción de los mensajes periodísticos por la población de los 50, 60 y 70}

El punto de partida de la investigación fue el análisis de los diarios seleccionados como parte del estudio hemerocrítico propuesto; en consecuencia, la técnica de las fuentes orales es la metodología que dirige este acápite a través de la extracción de información que proporcionaron informantes, pues es coherente otorgarles voz a las personas que vivieron dichos acontecimientos.

Ricoeur citado por Soní (2003) concibe a la interpretación como arte de descifrar los significados segundos del lenguaje simbólico y denota que el texto habla por sí mismo, y de éste surge la interpretación. En este sentido, es muy importante considerar los constructos sociales que estaban implantados en la sociedad nicaragüense de la época en 
estudio, a través de los cuales los lectores y las lectoras de las noticias e informaciones traídas en este análisis agregaron una interpretación. Dicha construcción de esquemas sociales estaba fuertemente implantada en las editoriales de los medios de comunicación.

Para entender la visión de las personas entrevistadas, se realizó una triangulación entre las categorías de análisis: Estereotipos de género y patrones de comportamientos, clases sociales y representaciones femeninas, y arquetipos de belleza y cultura femenina, con las variables sociodemográficas: sexo, religión, nivel académico y procedencia de cada informante.

En cuanto a la relación entre variable sexo y las categorías de análisis de la investigación, referente a la sumisión femenina de la época, una informante expresa: “[... ] solo su esposo, no traicionar con nadie a su esposo. Y yo soy un ejemplo... 59 años de matrimonio y solo conocí a ese señor... Pero como me enseñaron a ser una persona, tenía que hacer los quehaceres de la casa” (Coello 2016).

En cuanto a la categoría clases sociales, uno de los entrevistados argumenta un contexto negativo para las mujeres, pues además de su género otra desventaja era no pertenecer a la clase social alta o media alta: "Hay que ver que ambos periódicos (Novedades y La Prensa) desde el punto de vista ideológico sentaban a nivel general de la población la ideología de clase, de la clase dominante, es la clase que ostentaba el poder económico y político, era la burguesía... a la mujer solo le daban las páginas sociales, pero la mujer no tenía relevancia política en ese momento (Aguilera 2016).

La siguiente cita evidencia parte del relato de un hombre que evoca la percepción masculina en cuanto al comportamiento que debía asumir la mujer en la época:

Habían unas que se portaban bien, otras que se portaban mal... Cuando estaban con el marido ahí platicando de lo que ella hace, del asunto de los niños y todo eso que "te voy a cocinar tal cosa, que mañana voy a lavar la ropa". Cuando se portaba mal solo se peleaban que "vos andabas con otras mujeres y la casa me la has abandonado, que no me das para el sustento para la casa", y todo eso era pleito. (Rivera 2016) 
El elemento de belleza también estuvo interpretado de distintas maneras por cada persona como una manera de acertar a la subjetividad para concebirla y a la vez considerar las transformaciones que esta ha adquirido, haciendo hincapié entre lo tangible e intangible (Sandino 2016). Para Delgado (2016), el esquema de belleza en Nicaragua deriva de la cultura occidental, del modelo griego en cuanto a la fineza de los contornos.

En cuanto a la relación entre variable religión y las categorías de análisis de la investigación se destaca la legitimación religiosa de las alianzas entre hombre y mujer. De acuerdo con Delgado (2016), “ [...] la institución que socialmente legitimaba las relaciones de pareja era la institución religiosa traducida en lo que era el matrimonio eclesiástico; el Estado reconocía el matrimonio civil únicamente, no el eclesiástico, pero socialmente era bien visto el eclesiástico y no solo la unión civil”.

En la época en estudio, las mujeres tenían poco acceso a la educación, la concepción machista derivaba en limitaciones para que estas asistieran a escuelas o universidades y así lograran obtener un trabajo. Una informante recuerda:

...en aquel tiempo la mujer como que estaba pisoteada bajo el hombre, que solo el hombre es el que tenía el derecho de hacer muchas cosas... tal vez si una mujer quería estudiar, para qué va ir a estudiar, para qué va a ir a aprender algo si es solo para que esté cuidando los hijos, atendiendo al marido". (Parrales 2016)

Asimismo, las personas informantes concuerdan en que los protagonistas de los mensajes que emitían los medios de comunicación estaban ligados a una clase social definida: "en las páginas sociales solo la gente grande, pero una gente pobre no teníamos acceso" (Parrales 2016). El mismo marco figuraba para los concursos de belleza en los que la condición social adquirió gran valor a favor de las jóvenes participantes. Con esto coincide Sincleir (2016): “[...] y por lo general eran las hijas de los capitanes, de los doctores, todos los que tenían un estatus de vida bien formado". 


\section{Interpretación y síntesis de la visión de las personas informantes}

Las personas entrevistadas manifiestan que la relegación de las mujeres para designarles los roles hogareños eran condiciones orientadas por la sociedad, la religión y el núcleo familiar. Desde la visión particular de las mujeres, estas asumen por tradición el rol de ser fieles a su hogar y al marido. Por su parte, el hombre acepta que esta posición de machismo en todos los ámbitos obligaba a la sumisión de la mujer.

Algunos informantes, hombres y mujeres, coincidieron en que existen dos tipos de belleza: tangible e intangible, es decir, una belleza interna o moral y otra externa o visible. Las personas entrevistadas estiman que el valor asignado a la belleza en la época estaba legitimado a través de los concursos y eventos en los que solo participaban las jovencitas que cumplían con ciertas características físicas; estas debían ser de contextura proporcionada y no delgada. Los modelos de belleza se establecieron a través de la cultura norteamericana y europea.

Para concluir, se aborda un análisis con base en cada una de estas categorías y subcategorías. En primer lugar, el modelo que alude a la función femenina en la sociedad nicaragüense de los años 50, 60 y 70, prestablecía una mujer que debía mantenerse como base dentro de las familias y la sociedad. Ellas están llamadas a cuidar "su" hogar, marido e hijos. Este modelo está condicionado por el régimen imperante, estos son el gobierno, los medios de comunicación y la población en general, los cuales refuerzan estas conductas sociales, que se establecen como fenómenos culturales.

Las clases dominantes en las décadas de los años 50, 60 y 70 en Nicaragua -clase alta y media alta- se encuentran tangibles a través del lenguaje que los medios de comunicación escritos empleaban para designar un grado de relevancia a las mujeres y su entorno. Los temas de interés que estos difundían legitimaban las distintas acciones a las que estaban sujetas las mujeres a nivel social. Así, los espacios para ellas estaban reducidos a labores benéficas de carácter social y humanitario. De igual manera, los apellidos de las mujeres mencionados en las notas de carácter social de ambos periódicos, evidencian la inmersión de las clases sociales dominantes de aquel entonces, mientras las 
mujeres de clase baja carecían de oportunidades para desarrollarse en los ámbitos social y académico.

El ideal de familia fue un mecanismo para implantar la ideología de la responsabilidad matrimonial que carga la mujer, ella debía entablar una relación con miras a construir su familia a través del matrimonio, sistema instituido por la religión católica. El matrimonio civil estaba reconocido de manera legal, pero la unión eclesiástica era legitimada por la sociedad, ideología que fortalecieron los medios de comunicación.

Los indicios de insatisfacción de las mujeres revelan una clara alusión a la emancipación en rechazo a los esquemas culturales que se les habían adjudicado. Esta liberación se reflejó en el aspecto de la moda, autonomía de los cuerpos y su inserción en espacios laborales. Las décadas en estudio estuvieron marcadas por grandes cambios en el plano de la moda femenina, ésta constituida como una de las pautas que avizoraba la revolución sobre la visión de las mujeres en esos contextos. A través de distintos eventos como los certámenes de belleza, promociones de marcas, y figuras femeninas icónicas del cine y la moda, los medios de comunicación fortalecieron arquetipos en el imaginario colectivo de la sociedad nicaragüense de la época, provenientes de las sociedades estadounidenses y europeas.

La sociedad de ayer transmite a la actual algunos esquemas de vida que se han constituido como impedimentos para el desarrollo integral de la mujer; por ejemplo, la pervivencia de algunas conductas transferidas exclusivamente a las mujeres y otras en particular a los hombres referidas a roles sociales. Sin embargo, quienes vivieron aquella época, aunque recuerdan con nostalgia su pasado, consideran muy importantes los grandes avances de una nueva sociedad, construida con el protagonismo de las propias mujeres en su relación social hombre-mujer en contextos dentro del hogar y ámbitos profesionales, lo que ha permitido a las mujeres una autonomía económica y social.

La investigación de género como una categoría transdisciplinaria que desarrolla un enfoque globalizador y un importante avance en las ciencias sociales, debe abordarse desde distintos campos y disciplinas, puesto que aún existen muchos datos que auscultar los cuales aglomeran una gran relevancia a nivel político y social. Es importante destacar 
el estudio de la mujer desde los medios de comunicación como legitimadores de conductas sociales y patrones de comportamientos. Hoy día, las técnicas de estudio como el análisis hemerocrítico y la agenda Setting permiten estudiar el papel de estos en el contexto deseado. En síntesis, se pueden conjugar las técnicas de investigación a través de estudios interdisciplinarios, multidisciplinarios y transdisciplinarios, a fin de contribuir al avance de las ciencias sociales. Por último, la visión integradora u holística de esta investigación para establecer el tipo de representación de la mujer, implicó una novedad para el estudio con perspectiva de género; sin embargo, de acuerdo a los hallazgos se propone abrir, desde las ciencias sociales, un debate al tema de la belleza en la modernidad, puesto que se ha establecido algunas evoluciones en su construcción.

\section{Referencias bibliográficas}

Documentos físicos

Buttler, Judith. 1999. El género den disputa. Feminismo y la subversión de la identidad. Barcelona: Paidós.

Cobo del Arco, M. 2000. Políticas de género en la época liberal de Zelaya. Managua: UCA.

D Ádamo, Orlando, Virginia García y Flavia Freidenberg. 2007. Medios de comunicación y opinión publica. Madrid: McGraw-Hill/Interamericana de España.

De la Torre Villar, Ernesto y Ramiro Navarro de Anda. 1990. Metodología de la investigación, bibliográfica, archivista y documental. D.F. México: Mc.Graw-Hill.

Eco, Umberto. 2006. Historia de la belleza. Barcelona: Lumen.

Fraser, Ronald. 1993. "La historia oral como historia desde abajo". Ayer, nº12: 79-92.

Jónasdóttir, Anna G. 1993. El poder del amor ¿Le importa el sexo a la Democracia? Valencia: Ediciones Cátedra.

Olivera, Mercedes. 1992. El poder de las mujeres. Nicaragua: Cenzontle.

Morales, O. 2003. Fundamentos de la investigación documental y la monografía. Venezuela: Universidad de Los Andes.

Pereyra, Carlos et al. 2005. Historia ¿para qué? Siglo Veintiuno. D.F. México: México Editores 
Lagarde, Marcela. 1996. "El género, fragmento literal: La perspectiva de género". Género y feminismo. Desarrollo humano y democracia. Madrid: Horas y horas. 13-38.

Lamas, Marta. 1996. El género: La construcción cultural de la diferencia sexual. Coordinación de Humanidades, Programa Universitario de Estudios de Género PUEG, UNAM.

Montenegro, Rosario. 2012. "El voto femenino en Nicaragua: una historia oculta". Revista Encuentro, n'. 91 (2012): 91-115

Rodríguez, Ileana. 1990. Registradas en la Historia. 10 años del quehacer feminista en Nicaragua. Centro de Investigación y Acción para los Derechos de la Mujer CIAM Editorial Vanguardia.

Somoza, Anastasio. 1967. “...hacia la meta... "Mensajes políticos del General Anastasio Somoza Debayle, Presidente Constitucional de Nicaragua 1967-1972. Ante la Gran Convención Liberal Nacionalista y durante su Campaña Presidencial. Secretaría de Información y Prensa de la Presidencia de la República. Editorial San José-Managua.

Valdivieso, María I. et al. 2004. La historia de las mujeres: Una revisión historiográfica. Valladolid: Universidad de Valladolid.

Van Dijk, Teun. 1996. La noticia como discurso. Comprensión, estructura y producción de a información. Barcelona: Paidós.

Documentos electrónicos

Barthes, $\quad$ Roland. $1993 . \quad L a$ aventura semiológica. http://www.mercaba.org/SANLUIS/Filosofia/autores/Contempor\%C3\%A1nea/Ba rthes/La\%20aventura\%20semiol\%C3\%B3gica.pdf [2 junio abril 2016].

Chomsky, Noam. (s.f.): "Diez estrategias de manipulación mediática". http://www.revistacomunicar.com/pdf/noam-chomsky-la-manipulacion.pdf [2 mayo 2016].

Gómez, Eloy. (s.f) "El concepto de cultura". Creative Commons 3.0. Santader: Universidad de Cantabria. http://ocw.unican.es/humanidades/introduccion-a-laantropologia-social-y-cultural/material-de-clase-1/pdf/Tema1-antropologia.pdf [2 febrero 2016].

Robles, Claudio. 2012. "El concepto de familia y la formación académica en Trabajo Social". Revista Debate público, Reflexión de trabajo social. http://trabajosocial.sociales.uba.ar/web_revista_3/pdf/8_robles.pdf $[2$ febrero 2016]. 
Soní, Araceli. 2003. "Ricoeur y la teoría de la recepción". Anuario 2002. UAM-X. México. 385-399.: sotohttp://148.206.107.15/biblioteca_digital/capitulos/23594gpr.pdf [10 septiembre 2016].

UNICEF (s.f) ¿Te suena familiar? La familia que hemos construido. http://www.unicef.cl/centrodoc/tesuenafamiliar/06\%20Construido.pdf

La belleza para Platón. http://filosofia.laguia2000.com/filosofia-griega/la-belleza-paraplaton\#ixzz3nnKlYFBa [3 de mayo 2016].

La sociedad $y$ sus contextos. http://www.eumed.net/librosgratis/2012b/1191/concepto_de_sociedad.html [5 abril 2016].

¡Vaya historia de la minifalda! www.elnuevodiario.com.ni/actualidad/ 346956minifalda-vaya-historia/ [2 junio 2016]

"Los géneros en el fotoperiodismo". http://www.fotoperiodismo.org/fotografiadocumental.pdf [2 abril 2016].

"La fotografía como texto informativo". http://datateca.unad.edu.co/contenidos/401117/Contenidos_2015/Fotografia_com o_texto_informativo.pdf [2 abril 2016].

"El periodista sabe proponer infografías". http://www.revistalatinacs.org/067/cuadernos/28_infos.pdf [2 abril 2016].

\section{Fuentes hemerográficas}

\section{Diario Novedades}

Novedades, mes de junio del año 1950

Novedades, mes de abril de 1957

Novedades, mes de marzo del año 1969

Novedades, mes de mayo del año 1969

Novedades, mes de marzo del año 1977

Novedades, mes de junio del año 1977

Novedades, mes de febrero del año 1978 


\section{Diario La Prensa}

La Prensa, mes de abril del año 1955

La Prensa, mes de mayo del año 1955

La Prensa, mes de junio del año 1955

La Prensa, mes de mayo del año 1969

La Prensa, mes de marzo de 1970

La Prensa, mes de febrero del año 1979

\section{Relación de informantes}

Aguilar Guevara José Ramón (2016).

Entrevista realizada por Ruth Nohemí Rojas Icabalzeta, el 14 de julio de 2016.

Coello Orozco Sixta del Carmen (2016).

Entrevista realizada por Ruth Nohemí Rojas Icabalzeta, el 13 de julio de 2016.

Delgado Vanegas José Salomón (2016).

Entrevista realizada por Ruth Nohemí Rojas Icabalzeta, el 20 de julio de 2016.

Hernández López María Petrona (2016).

Entrevista realizada por Ruth Nohemí Rojas Icabalzeta, el 13 de julio de 2016.

Parrales Chavarría. Eva Apolonia (2016).

Entrevista realizada por Ruth Nohemí Rojas Icabalzeta, el 14 de julio de 2016.

Rivera Avellán José Miguel (2016).

Entrevista realizada por Ruth Nohemí Rojas Icabalzeta, el 16 de julio de 2016. 
Sandino Robleto Mariza de la Cruz (2016).

Entrevista realizada por Ruth Nohemí Rojas Icabalzeta, el 16 de julio de 2016.

Sincleir Mortty Steven Erick (2016).

Entrevista realizada por Ruth Nohemí Rojas Icabalzeta, el 16 de julio de 2016. 


\section{- Anexos}

Figura 1

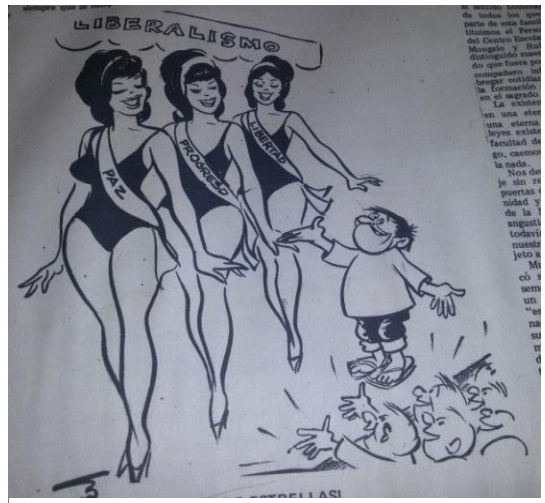

Novedades, 4 de mayo, 1969

Figura 3

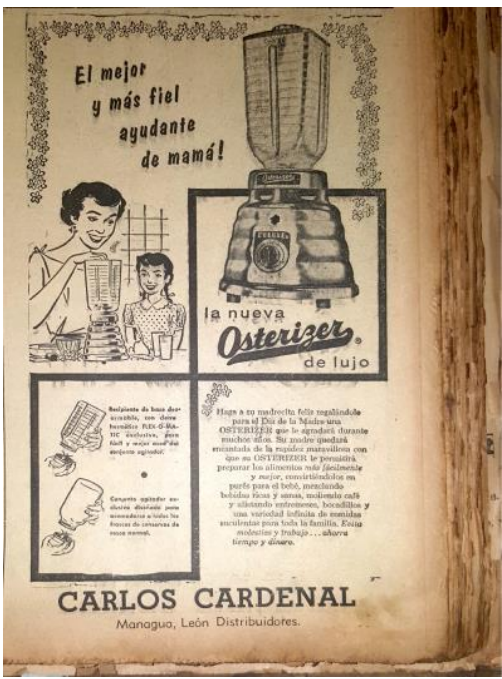

La Prensa, 12 de mayo, 1955
Figura 2

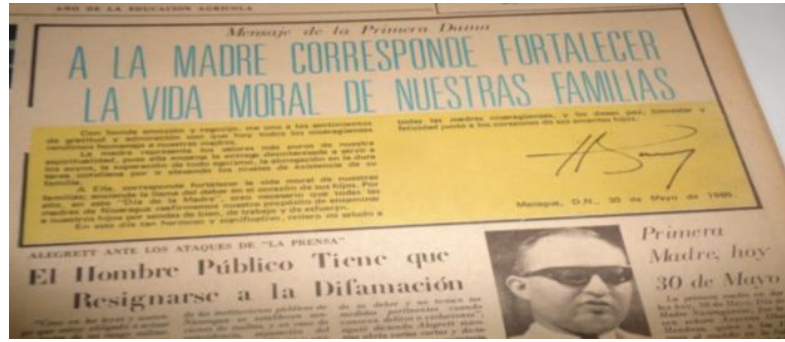

Figura 4

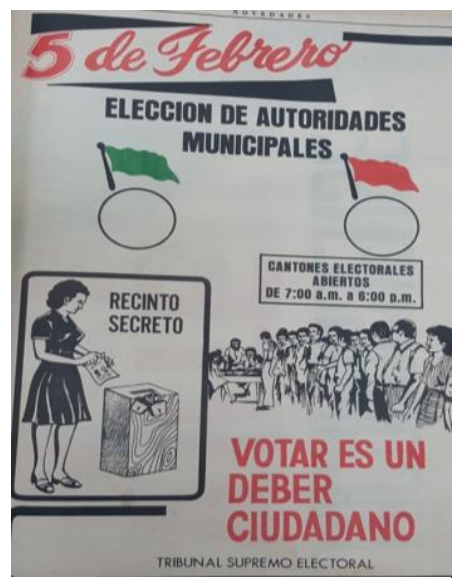

Novedades, 4 de febrero, 1978

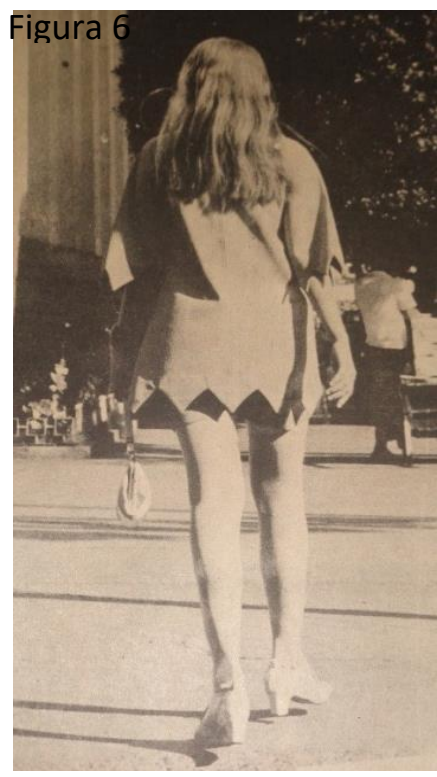

Novedades, 3 de enero, 1969
Figura 5

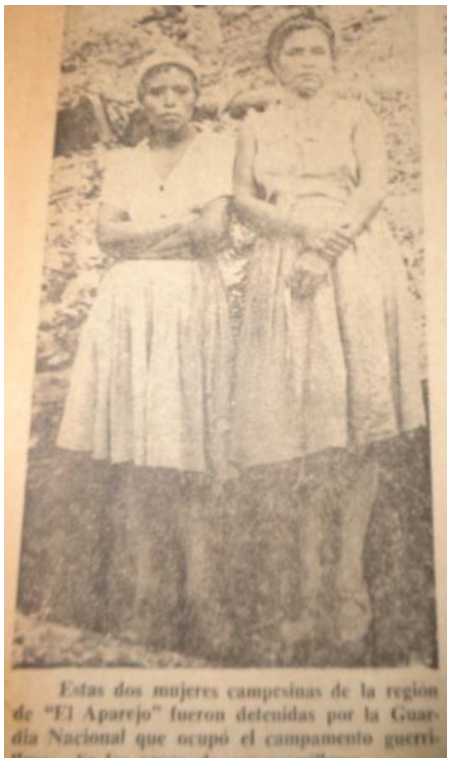

La Prensa, 1 de marzo, 1979

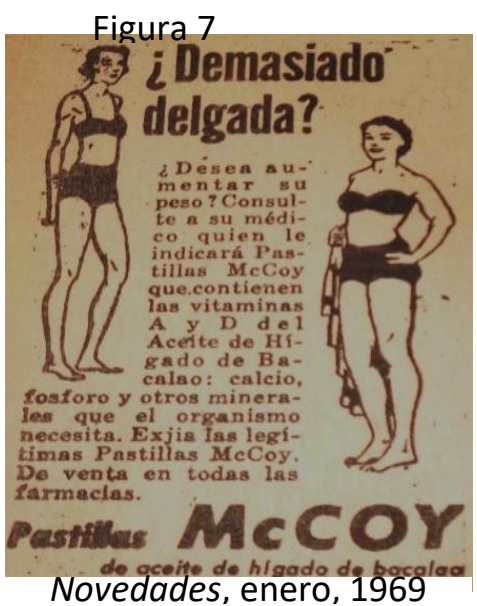

